P0-0923 VARICOCELE TREATMENT IN PAEDIATRIC AGE: REPORT OF DIFFERENT TECHNIQUES

${ }^{1} \mathrm{~N}$ Zampieri, ${ }^{1} \mathrm{~A}$ Mantovani, ${ }^{1} \mathrm{G}$ Scirè, ${ }^{1} \mathrm{~S}$ Zambaldo, ${ }^{2} \mathrm{G}$ Zampieri, ${ }^{1} \mathrm{FS}$ Camoglio. ${ }^{1}$ Department of Surgical Sciences Pediatric Surgical Unit University of Verona Policlinico G. B. Rossi Piazzale L. A. Scuro N.1 37134 Verona Italy, University of Verona, Verona, Italy; ' Department of Surgery, CASA Di Cura San Francesco, Verona, Italy

10.1136/archdischild-2014-307384.1542

Background Varicocele is the first andrological disease in adolescent male. The aim of this study is to report our experience in varicocele treatment in paediatric age.

Materials and methods We analysed patients treated with different techniques between January 2005 and January 2013. Inclusion criteria for the study were created. Patients were treated with different techniques based on the type of spermatic vein reflux detected on Doppler velocimetry. Type I spermatic vein reflux mini-invasive approaches, type II and III vein reflux open approaches. The following rates were considered as study endpoints: hydrocele rate; rate of relapses; rate of persistence; rate of testicular atrophy.

Results During the study period 522 surgical varicocelectomies were performed. 345 cases were treated with videolaparoscopy (66,1\%), 115 cases with an open inguinal technique (22,1\%), 47 cases $(9 \%)$ received a subinguinal technique (trans-scrotal technique in 18 cases and subinguinal technique in 29 cases) and 15 cases $(2,8 \%)$ were treated with the one-trocar transumbilical technique.

The laparoscopic technique and the subinguinal techniques showed a lower rate of hydrocele. Laparoscopic technique had less rate of relapses.

Conclusions The laparoscopic techniques had less rate of relapses respect to other technique; this results could be related to the technique chosen respect to the type of spermatic vein reflux.

\section{PO-0924 ELASTOSONOGRAPHY OF THE CORPUS SPONGIOSUM: COMPARISON BETWEEN NORMAL AND HYPOSPADIC ANATOMY}

${ }^{1} \mathrm{~N}$ Zampieri, ${ }^{2} \mathrm{C}$ Bruno, ${ }^{1} \mathrm{~A}$ Mantovani, ${ }^{1} \mathrm{G}$ Scirè, ${ }^{1} \mathrm{~S}$ Zambaldo, ${ }^{1} \mathrm{FS}$ Camoglio. ${ }^{1}$ Department of Surgical Sciences Pediatric Surgical Unit University of Verona Policlinico G.B. Rossi Piazzale L.A. Scuro N.1 37134 Verona Italy, University of Verona, Verona, Italy; ${ }^{2}$ Department of Radiology, University of Verona, Verona, Italy

\subsection{6/archdischild-2014-307384.1543}

Background Elastosonography analyses the variable elasticity of a tissue by recording the changes of RF pulses from a structure, before and after manual compression.

The aim of this study is to apply the elastosonography on normal and hypospadic penis to verify the structural differences in tissues composition and stiffness.

Materials and methods 28 subjects were enrolled, mean age 24 months: 17 patients on post-operative follow-up for distal hypospadia and 11 age-matched subjects as control group. Elastosonography recorded antero-posterior and transverse diameters of the cavernous corpora, urethral and corpus spongiosum diameters, elastographic index of elasticity of the corpus spongiosum.
The latter was defined as soft, medium-hard or hard. We assigned the value 1 to soft tissue, 2 and 3 to medium-hard and hard respectively.

All scan were performed at the base of the penis, as far as possible from the region involved in the surgical procedure.

Results Average antero-posterior and transverse diameters of the cavernous corpora were $4.2 \mathrm{~mm}$ (DS 0.83 ) and $6.5 \mathrm{~mm}$ (DS 1.12) for hypospadic group and $7.3 \mathrm{~mm}$ (DS 1.28) and $9.0 \mathrm{~mm}$ (DS 1.67) for control group. These data showed statistically significant difference between the two groups for both the diameters $(\mathrm{p}<0.05)$.

Corpus spongiosum diameter was 1.9 (DS 0.33) for hypospadic group and $2.7 \mathrm{~mm}$ (DS 0.73) for control group ( $<<0.05$ ).

Elastography showed a corpus spongiosum stiffness defined as medium-hard or hard in all cases of the pathologic group and soft in all the subjects of the control group $(\mathrm{p}<0.05)$.

No significant difference was found for urethra diameter between the two groups.

Conclusions Elastosonography showed how the hypospadia anatomy is deeply altered, even in an anatomical area far from meatal abnormality: corpus spongiosum in hypospadic penis seems to be globally stiffer and less elastic.

Moreover, cavernous corpora are less developed.

These preliminary results suggest the feasibility of the elastosonography for the evaluation of hypospadic penis. It could be useful during surgical plan to have a better definition of the calibres and quality of the tissues, especially in proximal hypospadia.

We believe that this methodic could also be important during the early post-operative follow-up.

\section{Paediatric Surgery}

\section{PO-0924a ROLE OF JNK IN THE TREATMENT OF INTESTINAL TREFOIL FACTOR FOR NECROTIZING ENTEROCOLITIS IN RATS}

1J Zhao, ${ }^{2} X$ Fu. ${ }^{1}$ Department of Pediatric Surgery, The First Hospital of Jilin University, Changchun, China; ${ }^{2}$ Nursing Department, The Third Hospital of Jilin University, Changchun, China

\subsection{6/archdischild-2014-307384.1544}

Purpose To explore the role of JNK in the treatment of intestinal trefoil factor for necrotizing enterocolitis in rats

Methods Thirty wistar rats, one day after birth, were equally divided into three groups: normal control group, NEC model group, and NEC+ITF group. The rats were fed for 3 days and killed on the 4th day. The ileocecum were obtained for histological examination by HE staining, JNK protein expression detection by Western-Blot and JNK gene transcripts detection by RT-PCR.

Results The level of JNK protein expression and gene transcripts in NEC model group was higher than that in normal control group. Compared with NEC Model group, the level of JNK expression and gene transcripts was obviously decreased in NEC + ITF group. 